\title{
Increased hGH Production Rate after Low-Dose Estrogen Therapy in Prepubertal Girls with Turner's Syndrome
}

\author{
NELLY MAURAS, ALAN D. ROGOL, AND JOHANNES D. VELDHUIS \\ Nemours Children's Clinic, Jacksonville, Florida 32247 [N.M.], Departments of Pediatrics and Pharmacology \\ [A.D.R]., and Department of Internal Medicine [J.D.V.] University of Virginia, Charlottesville, Virginia 22908
}

\begin{abstract}
Low-dose estrogen therapy significantly increases radioimmunoassayable serum hGH concentrations in the prepubertal hypogonadal female. In this study, we have examined the effects of short- and long-term low-dose ethinyl estradiol therapy on the endogenous production rates and metabolic clearance rates of hGH. We used deconvolution mathematical modeling to provide quantitative estimates of individual secretory parameters and to calculate subject-specific hGH metabolic clearance rates, by using all serum hGH concentrations and their variances considered simultaneously. Nine girls with Turner's syndrome (mean age $7.7 \pm 0.5 \mathrm{y}$ ) were studied on three separate nights by drawing blood every $20 \mathrm{~min}$ from 2200 to $0800 \mathrm{~h}$ before (I), after $1 \mathrm{wk}$ (II), and 5 wk (III) of 100 $\mathrm{ng} / \mathrm{kg} / \mathrm{d}$ ethinyl estradiol therapy orally. We found that the endogenous hGH production rate more than doubled in all patients studied after 5 wk of ethinyl estradiol therapy (194 \pm 22 (I), $290 \pm 43$ (II), and $412 \pm 66$ (III) $\mu \mathrm{g} / \mathrm{L} / 12$ h; $p<0.05$ for I and III). The half-life of endogenous hGH was not altered in the estrogen treatment paradigm with a mean of $19 \pm 1.6 \mathrm{~min}$ in study $I$ and $18 \pm 1.2 \mathrm{~min}$ in both studies II and III. Our results suggest that even prepubertal concentrations of gonadal steroids in the hypogonadal female may be physiologically relevant to the maintenance of normal somatotrope secretory function. (Pediatr Res 28 : 626-630, 1990)
\end{abstract}

\section{Abbreviations}

EE, ethinyl estradiol

GHRH, growth hormone-releasing hormone

hGH secretion is significantly influenced by sex steroid hormones. For example, studies have shown that circulating hGH concentrations increase under normal physiologic circumstances such as during puberty and in response to the exogenous administration of androgens (1). Recently, we demonstrated significant increases in hGH concentrations after the administration of low doses of estrogen to a group of hypogonadal girls with Turner's syndrome (2). Other investigators have also found a marked stimulative effect of sex steroid hormones on hGH levels (3) and on linear growth $(4,5)$. Whether increased serum hGH levels were due to increased production or decreased clearance of hGH could not be ascertained from these studies.

Received January 22, 1990; accepted July 11, 1990.

Correspondence: Nelly Mauras, M.D., Nemours Children's Clinic, P. O. Box 5720 , Jacksonville, FL 32247.

Supported by the Nemours Foundation (Jacksonville, Florida) and NIH General Clinical Research Grant RR-00847 (UVA).
New deconvolution techniques allow the precise and valid assessment of alterations in the amplitude, duration, and frequency of pulsatile hormone secretion, which give rise to the varying plasma hormone concentration profiles. For example, a multiple parameter deconvolution model $(6,7)$ allows one to evaluate quantitatively the number of spontaneous hGH secretory bursts per $24 \mathrm{~h}$, as well as their amplitude, frequency, duration and hGH mass secreted per burst. Thus, subject-specific production and metabolic clearance rates of endogenous hGH can be estimated with this model. However, to date, a comprehensive assessment of whether changes in hGH production and/ or clearance rates are elicited by sex steroid hormones has not been accomplished, particularly in children with gonadal failure. Our study was designed to characterize changes in hGH production and metabolic clearance rates induced by the administration of low-dose estrogen in the hypogonadal prepubertal female.

\section{MATERIALS AND METHODS}

Study design. Nine young girls with Turner's syndrome participated in our study after informed written consent was obtained from their parents. Our study was approved by the Jacksonville Baptist Medical Center Investigational Review Board. Seven of these nine subjects were reported previously (2). Their ages ranged between 5.4 and 10.4 y (mean $7.7 \pm 0.5 \mathrm{y}$ ) and all had $45, \mathrm{XO}$ or related karyotypes. None had received hormonal therapy previously and all were in good health. Each patient was admitted on three separate nights to the hospital-based research unit of the Nemours Children's Clinic. At baseline a heparin lock needle was inserted in a forearm vein for frequent blood sampling. From 2000 to $0800 \mathrm{~h}$, blood was withdrawn at 20 -min intervals for serum hGH determinations. Four wk after discharge, all girls were started on $100 \mathrm{ng} / \mathrm{kg}$ of EE orally daily. One wk (study II) and 5 wk (study III) after initiation of the lowdose EE therapy, the patients returned for an identical study.

Assays. Serum hGH concentrations were measured in duplicate using the Nichols Institute hGH immunoradiometric assay kits (San Juan Capistrano, CA). The intraassay coefficient of variation was $<8 \%$ with a sensitivity of $0.5 \mu \mathrm{g} / \mathrm{L}$.

Data analysis. The concentration of a hormone in the blood compartment at any time derives from the combined effects of simultaneously occurring hormone secretion and hormone elimination or clearance. The secretory function (which denotes the secretory rate as a function of time) was constructed as a finite series of discrete secretory bursts, with determinable, statistically bounded amplitudes, half durations, and secretory times distributed randomly throughout the sampling interval $(6,7)$. A distinct secretory burst was then defined as a random (Gaussian) distribution of instantaneous molecular secretory rates (7) whose fitted amplitudes could be distinguished from zero (pure noise) at $p<$ 0.05 . The integral of these secretion pulses gives an ascending 
curve. This is illustrated algebraically as:

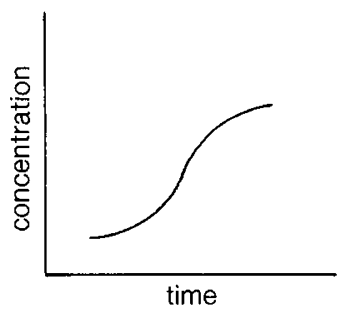

The elimination or clearance function was defined as a onecomponent exponential model with a subject-specific half-life of hormone elimination fitted simultaneously with the secretion parameters. This is illustrated algebraically as:

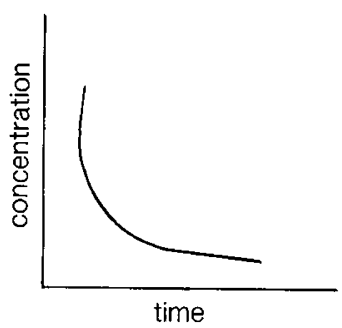

$E(t-z)$

elimination function

Because these two functions (secretion and clearance) act simultaneously to influence the same system, they are said to be mathematically "convolved." The serum hormone concentration resulting from the interaction of these two processes can be then described by a convolution integral illustrated here:
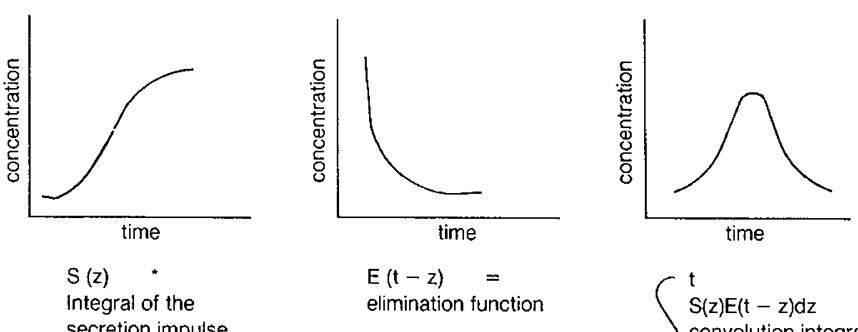

secretion impulse

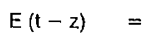

elimination function

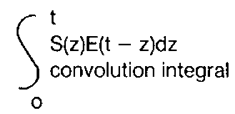

Superimposed upon this are the additional effects of such unpredictable variables as assay noise and experimental error, to produce the hormone concentration profiles we observed experimentally. The process of unraveling this convolution integral to uncover and describe these two separate convolved functions is referred to as "deconvolution." We assume in this model that the distribution volume and elimination rate constants in any one subject are invariant during the particular interval of observation (8). hGH concentrations were assumed to decay with hGH IRMA.

Estimating secretion and clearance parameters. We used a modified Gauss-Newton quadratic convergence procedure to determine the following individual parameter values and their joint statistical confidence limits: half-duration of each subject's secretory bursts; amplitudes of all secretory bursts; temporal positions of all secretory bursts; and single-component (nonexponential) subject-specific disappearance rate constants. The mass of hGH secreted per pulse is the integral (area) of the resolved secretory burst $(6,7)$, and the 12 -h production rate is the product of the total number of secretory bursts and the mean mass of hGH released per burst.

Previously, we have used cluster analysis (9) on the hGH concentration series to examine hormone "pulses." Using this procedure, one can estimate the frequency, amplitude, mean hormone concentration, and area under the curve of discrete $\mathrm{hGH}$ concentration measurements arranged by the time of the day. Secretion is not estimated by this method. Figure 1 com-
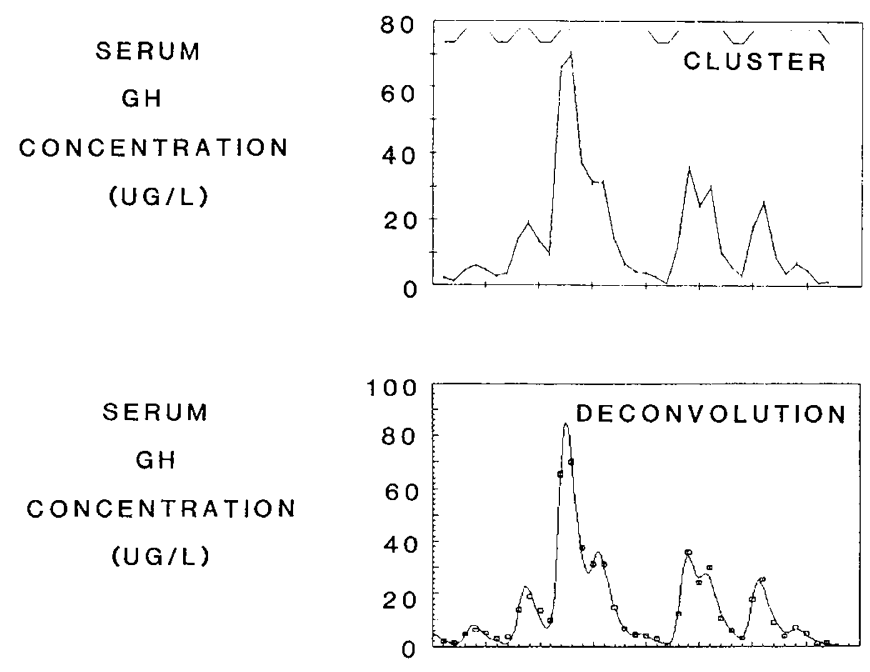

$$
\begin{gathered}
\text { GH } \\
\text { SECRETORY } \\
\text { RATE } \\
\text { (UG/L/MIN) }
\end{gathered}
$$

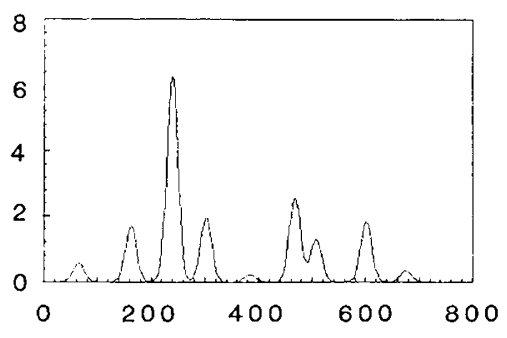

TIME (MIN)

Fig. 1. Graphic representation of the analysis of a serum hGH concentration series as analyzed by cluster analysis and deconvolution modeling. The top panel shows the changes in hGH concentrations over time. Deflections at the top represent the concentration pulses detected by cluster analysis. The middle panel shows a continuous curve representing hGH concentrations that would be expected to exist given the discrete pituitary episodes predicted. The bottom panel represents the somatotrope's secretory episodes as estimated by deconvolution, each as a Gaussian distribution of secretory rates.

pared the information obtained using this model with deconvolution. The top panel shows the analysis of hGH concentration series using cluster.

The middle panel shows a continuous curve representing $\mathrm{hGH}$ concentrations that would be expected to exist if the discrete pituitary episodes had occurred as predicted. This is the "reconvolution" curve, which allows the investigator to directly visualize how well the model describes the actual measured hormone concentrations. This curve provides a check on the reasonableness of the estimated parameters. In the bottom panel, the pituitary hGH secretion episodes estimated by deconvolution are illustrated graphically. Each burst is a Gaussian distribution of secretory rates and is separated from others by periods of secretory quiescence.

Statistics. Because of departures from normality, hGH secretory and clearance values were subjected to logarithmic transformation before application of Dunnett's test (10) for multiple protected comparisons with a single mean.

\section{RESULTS}

The deconvolution data for all nine patients studied are summarized in Table 1. For comparison, the cluster analysis of the $\mathrm{hGH}$ series on all nine patients is provided in Table 2 . Cluster data of seven of the nine patients were previously reported (1). 
Table 1. Deconvolution data summary*

\begin{tabular}{lccc}
\hline & Study I & Study II & Study III \\
\hline hGH half-life (min) & $19 \pm 1.6$ & $18 \pm 1.2 \dagger$ & $18 \pm 1.2 \dagger$ \\
hGH secretory burst half dura- & $27 \pm 2.3$ & $27 \pm 2.5 \dagger$ & $29 \pm 2.0 \dagger$ \\
$\quad$ tion (min) & & & \\
hGH secretory burst frequency & $5.3 \pm 0.6$ & $6.9 \pm 0.3 \dagger$ & $7.9 \pm 0.5 \ddagger$ \\
$\quad$ (no. bursts/12 h) & & & \\
$\begin{array}{l}\text { Interpulse interval (min) } \\
\text { Maximal rate of hGH release }\end{array}$ & $131 \pm 9.0$ & $105 \pm 3.8 \ddagger$ & $94 \pm 5.5 \ddagger$ \\
$\quad(\mu \mathrm{g} / \mathrm{L} \cdot \min )$ & $1.4 \pm 0.1$ & $1.5 \pm 0.2 \dagger$ & $1.8 \pm 0.3 \dagger$ \\
hGH mass/burst $(\mu \mathrm{g} / \mathrm{L})$ & $39 \pm 4.4$ & $43 \pm 6.1 \dagger$ & $51 \pm 5.8 \dagger$ \\
hGH production rate & $194 \pm 22$ & $290 \pm 43 \dagger$ & $412 \pm 66$
\end{tabular}

$(\mu \mathrm{g} / \mathrm{L} / 12 \mathrm{~h})$

* Data are means \pm SEM ( $n=9$ subjects in each study). The volume term (L) refers to subject-specific unit distribution volume for hGH.

$\dagger$ Not significant as compared to baseline by Dunnett's test. $\ddagger p<0.05$.

Table 2. hGH pulse analysis by cluster*

\begin{tabular}{|c|c|c|c|}
\hline & I & II & III \\
\hline $\begin{array}{l}\text { Mean hGH concentration } \\
(\mu \mathrm{g} / \mathrm{L})\end{array}$ & $6.7 \pm 0.9$ & $10.9 \pm 1.8$ & $12.8 \pm 1.5 \dagger$ \\
\hline $\begin{array}{l}\text { Mean area under pulse } \\
(\mu \mathrm{g} / \mathrm{L} \cdot \mathrm{min})\end{array}$ & \pm 43 & $958 \pm 135$ & $1190 \pm 229 \dagger$ \\
\hline $\begin{array}{l}\text { Mean pulse amplitude } \\
(\mu \mathrm{g} / \mathrm{L})\end{array}$ & $14.1 \pm 1.7$ & $22.3 \pm 3.4$ & $29.6 \pm 5.2 \dagger$ \\
\hline $\begin{array}{l}\text { Mean frequency } \\
\text { (pulses } / 12 \mathrm{~h})\end{array}$ & $4.9 \pm 0.5$ & $5.3 \pm 0.4$ & $5.8 \pm 0.5$ \\
\hline
\end{tabular}

* Data expressed as mean \pm SEM. Cluster analysis estimates serum concentration peaks rather than secretion pulses. The latter are presented in Table 1.

$\dagger p<0.05$ (III $v s \mathrm{I})$.

Deconvolution analysis provides an estimate of the subjectspecific half-life of hGH disappearance from plasma. The halflife of endogenous hGH was not altered in the estrogen treatment paradigm with a mean of $19 \pm 1.6 \mathrm{~min}$ in study $\mathrm{I}$ and $18 \pm 1.2$ min in both studies II and III.

This analysis also provides an estimate of the duration of $\mathrm{hGH}$ secretion associated with each secretory burst. The half-duration (time elapsing at half-maximal amplitude) of computer-detected hGH secretory bursts was stable over the two estrogen-treatment visits. ( $27 \pm 2.3$ min study I versus $27 \pm 2.5$ min study II and 29 \pm 1.2 min study III).

As shown in Figure 2, when deconvolution analysis was applied to the hGH concentration series, the frequency of the hGH secretory bursts (number of hGH secretory bursts/12 h) increased after the second treatment visit $(5.3 \pm 0.6$ study I, $6.9 \pm 0.3$ study II, and $7.9 \pm 0.5$ study III). Conversely, the interpulse interval decreased on the first and second treatment visit (Fig. 3 ). The mass of hGH released per secretory burst as well as the amplitude of the hGH burst (maximal rate of hGH release) increased by the third treatment visit, but the latter change did not achieve statistical significance.

The production rate of endogenous $\mathrm{hGH}$ can be calculated as the product of the number of hGH secretory bursts and the mass of hGH secreted per burst. Assuming a correct estimate of halflife (above), such production rates accurately reflect the effect of subject-specific metabolic clearance rates acting upon all secreted hormone molecules (4). During the baseline study, the hGH production rate was $194 \pm 22$ versus $290 \pm 43 \mu \mathrm{g} / \mathrm{L} / 12 \mathrm{~h}$ during study II and $412 \pm 66$ during study III $(p<0.05$, Fig. 4$)$.

An example of the deconvolution fitted serum hGH profiles in two patients is shown in Figures 5 and 6, comparing the changes during the three study nights for estimated serum hGH concentrations as well as calculated hGH secretion rate.

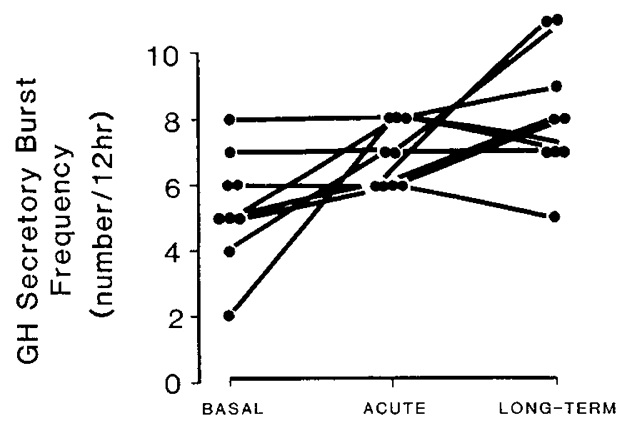

Fig. 2. Subject-specific frequencies of pulsatile hGH secretion in nine patients with Turner's syndrome at baseline, after $1 \mathrm{wk}$ (acute), and after 5 wk (chronic) of therapy with $100 \mathrm{ng} / \mathrm{kg}$ of EE orally. The $12-\mathrm{h} \mathrm{hGH}$ concentration series were collected by drawing blood at 20 -min intervals through an indwelling catheter from 2000 to $0800 \mathrm{~h}$ and analyzed by deconvolution methods. hGH secretory burst frequencies (numbers of $\mathrm{hGH}$ bursts $/ 12 \mathrm{~h}$ ) before and after long-term therapy were statistically significant $(p<0.05)$.

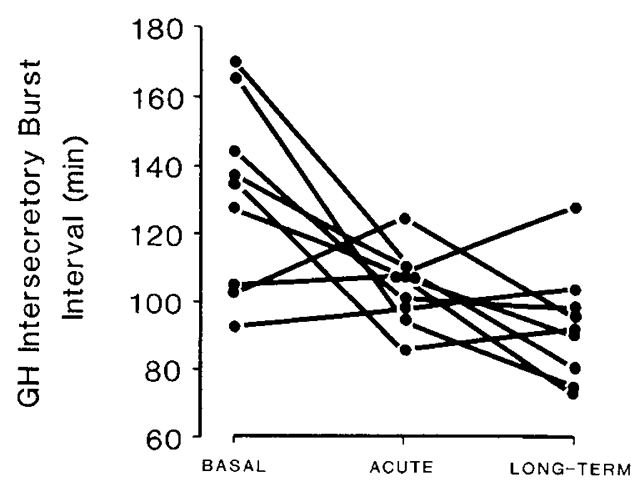

Fig. 3. hGH intersecretory burst interval ( $\mathrm{min}$ ) or the mean time in min elapsing between consecutive secretory burst centers. The individual subject data are shown. ( $p<0.05$ compared to baseline).

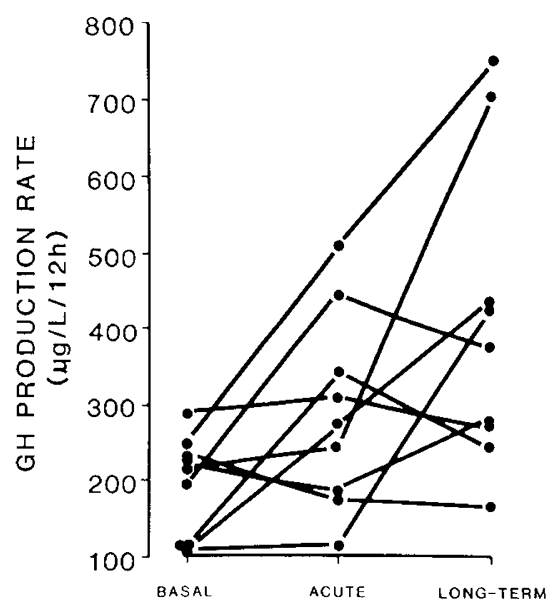

Fig. 4. Endogenous rate of hGH production in the patients basally and during treatment with low-dose estrogen. The individual subjectspecific nocturnal endogenous production rates of $h \mathrm{GH}$ were determined by deconvolution. The hGH production rate was significantly higher in the long-term treated group $(p<0.05)$. Data are expressed as mass units $(\mu \mathrm{g})$ per unit distribution volume $(\mathrm{L})$ per $12 \mathrm{~h}$.

\section{DISCUSSION}

Our studies demonstrate that the marked augmentation in the radioimmunoassayable $\mathrm{hGH}$ concentrations that we previously 

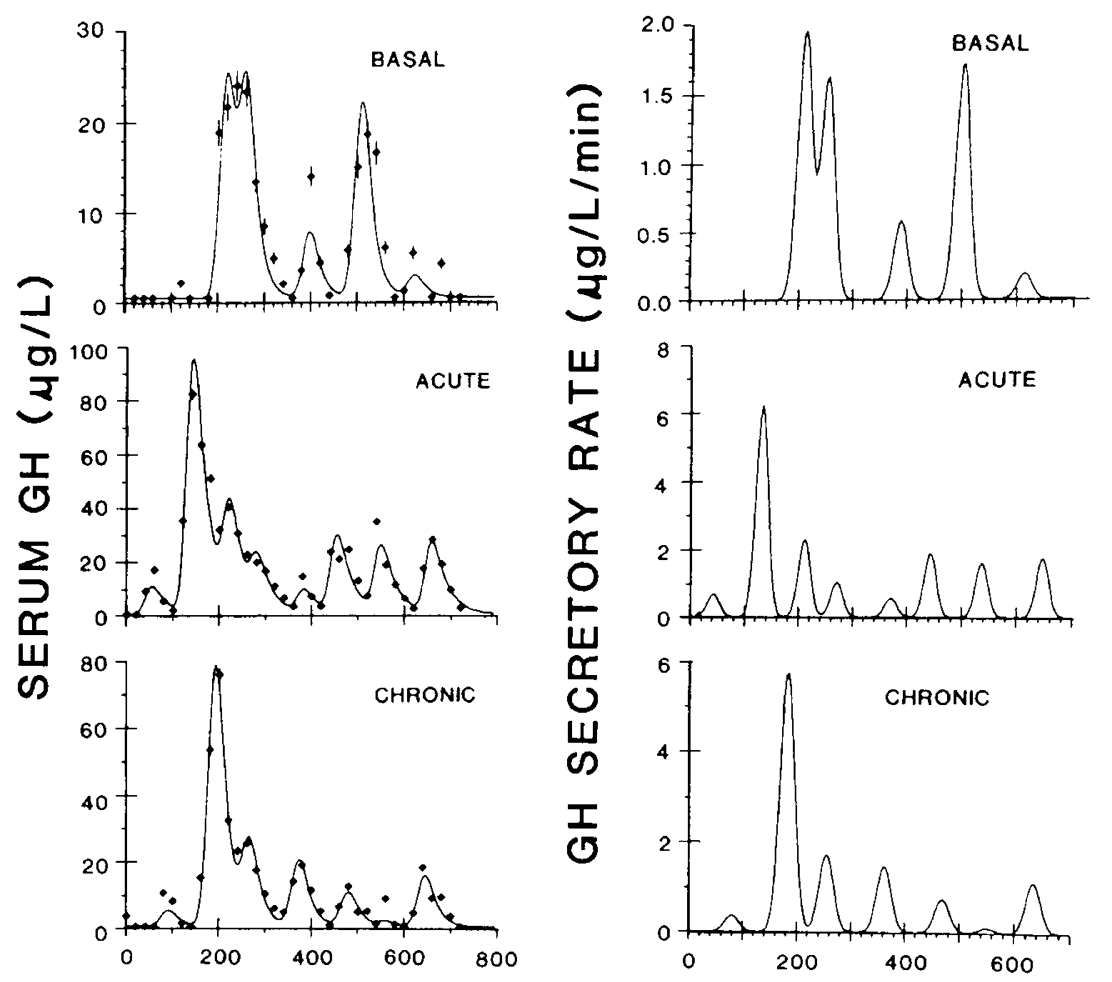

\section{TIME (min)}

Fig. 5. Illustrative profiles of pulsatile hGH concentrations and deconvolution-resolved hGH secretory rates in two patients with Turner's syndrome at baseline and during acute ( $1 \mathrm{wk}$ ) and chronic (5 wk) therapy with $100 \mathrm{ng} / \mathrm{kg} / \mathrm{d}$ of EE orally. The panels on the left give the observed serum hGH concentrations over time with the continuous fitted curves predicted by the deconvolution model. The panels on the right depict the computer-resolved hGH secretory rate plotted as a function of time, i.e. the hGH secretory profile devoid of subject-specific clearance. Note the differences in the ordinate scales. Time 0 represents $2000 \mathrm{~h}$.
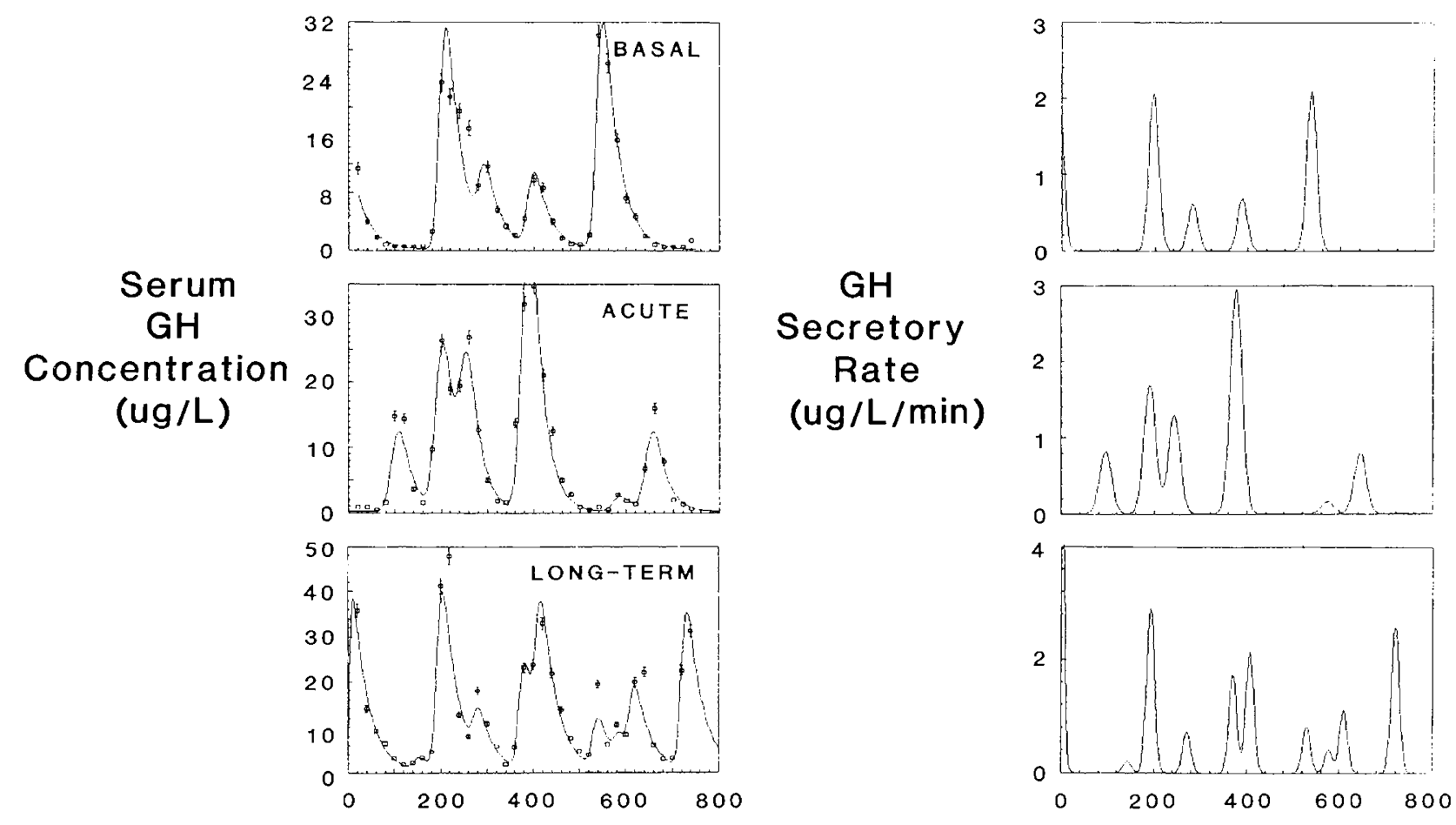

Time (min)

Fig. 6. The same data shown in Figure 5 for another subject. 
reported in girls with Turner's syndrome given low doses of estrogen (2) results from a significant increase in the hGH production rate rather than any change in hGH half-life (and therefore metabolic clearance rate) in the estrogen-enriched milieu. By deconvolution analysis, we assessed the subject-specific quantitative features of endogenous hGH secretion and metabolic clearance simultaneously, and observed that the nocturnal production rate of hGH after 5 wk of low-dose EE more than doubled in these patients.

Our previous observations (2) showed a remarkable increase in radioimmunoassayable serum hGH concentrations, with a corresponding decrease in serum FSH concentrations in a group of patients with Turner's syndrome after 1 and 5 wk of EE therapy. The previous analysis did not provide information regarding hormonal clearance and secretion. Deconvolution analysis on the other hand, allows the estimation of both secretory and clearance parameters. Therefore, the apparent increase in the frequency of hGH bursts appears to be accounted for by a better delineation of the hGH secretory event per se, in which deconvolution analysis was able to identify "pulses within pulses" of hGH activity. As can be seen in the reconvolution curves, they very closely approximate the known hGH concentrations of these patients. Similar approximations were obtained in all data sets. The additional deconvolution results shown in Figures 5 and 6 do not illustrate actual measured hGH concentrations, but rather estimated hGH secretory events (bursts).

The subject-specific half-lives of plasma $\mathrm{hGH}$ disappearance did not change during low-dose estrogen therapy. Specifically, the calculated half-life of endogenous hGH was 19 min before and $18 \mathrm{~min}$ after both estrogen treatment visits. These estimates are indistinguishable from those reported for endogenous hGH in normal men, whose hGH disappearance kinetics were investigated by the consecutive administration of hGH-releasing hormone and somatostatin (11). This failure of steroids to modify hGH metabolic clearance in vivo is important to note in view of the recent finding that $\mathrm{hGH}$ clearance rates can change under other conditions (12).

The precise neuroendocrine mechanisms subserving an increased hGH production rate after therapy with low doses of estrogen are not known, but the marked augmentation of the hGH secretory rate suggests a significant enhancement of somatotrope responsiveness to GHRH action and/or increased GHRH release. Such effects could be secondary to direct facilitative actions of low-dose estrogen on the somatotrope, decreased somatostatin inhibitory tone, and/or amplified GHRH pulse generation. Facilitation of GHRH action could elicit measureable increases in hGH secretion, which might otherwise have been undetectable, therefore causing an evident change in pulse frequency.
In conclusion, because even very low doses of estrogen can amplify pulsatile $\mathrm{hGH}$ secretion selectively without altering the $\mathrm{hGH}$ clearance, our data support the concept that prepubertal concentrations of gonadal steroids in the female may be important for the maintenance of normal somatotrope secretory function.

Acknowledgments. The authors thank Peggy Bowen, R.N., for her excellent assistance; Paula P. Azimi for computer analysis and William Tucker for art work; Sharon Treakle and the expert nursing staff of Jacksonville Wolfson Children's Hospital for their dedicated care of our patients; Cathrine Kern and Ginger Bauler for the running of the RIA; Dr. Robert Blizzard for his careful review of this manuscript; and Diane Goin for the excellent typing of this paper.

\section{REFERENCES}

1. Mauras N, Blizzard RM, Link K, Johnson ML, Rogol AD, Veldhuis JD 1987 Augmentation of growth hormone secretion during puberty: evidence for pulse amplitude-modulated phenomenon. J Clin Endocrinol Metab 64:596601

2. Mauras N, Rogol AD, Veldhuis JD 1989 Specific, time dependent actions of low-dose ethinyl estradiol administration on the episodic release of growth hormone, follicle stimulating hormone and luteinizing hormone in prepubertal girls with Turner's Syndrome. J Clin Endocrinol Metab 69:1053-1059

3. Moll GH, Rosenfield RL, Fang VS 1986 Administration of low-dose estrogen rapidly and directly stimulates growth hormone production. Am J Dis Child 140:124-127

4. Levine-Ross J, Cassorla FG, Skerda MC, Valk IM, Loriaux DL, Cutler GB 1983 A preliminary study on the effect of estrogen dose on growth in Turner's Syndrome. N Engl J Med 305:1104-1110

5. Levine-Ross J, Myerson Long L, Skerda M 1986 Effect of low-doses of estradiol on 6 month growth rates and predicted height in patients with Turner's Syndrome. J Pediatr 109:950-953

6. Urban RJ, Evans WS, Rogol AD, Kaiser DL, Johnson ML, Veldhuis JD 1988 Contemporary aspects of discrete peak detection algorithms: I. The paradigm of the luteinizing hormone pulse signal in men. Endocr Rev 9:3-37

7. Veldhuis JD, Carlson ML, Johnson ML 1987 The pituitary gland secretes in bursts: appraising the nature of glandular secretory impulses by simultaneous multiple-parameter deconvolution of plasma hormone concentrations. Proc Natl Acad Sci USA 84:7686-7690

8. Veldhuis JD, Iranmanesh A, Lizarralde G, Johnson ML 1989 Amplitude modulation of a burst-like mode of cortisol secretion subserves the circadian glucorticoid rhythm. Am J Physiol 257:E6-E14

9. Veldhuis JD, Johnson ML 1986 Cluster analysis: a simple, versatile, and robust algorithm for endocrine pulse detection. Am J Physiol 250:E486-493

10. Dunnett CW 1964 New tables for multiple comparisons with a control Biometrics 20:482-491

11. Faria ACS, Veldhuis JD, Thorner MO, Vance ML 1989 Half-time of endogenous growth hormone disappearance in normal man after stimulation of GH secretion by GHRH and suppression with somatostatin: studies with an in vivo clamp of the human somatotrope. J Clin Endocrinol Metab 68:535541

12. Rosenbaum M, Gertner JM 1989 Metabolic clearance rates of synthetic human growth hormone in children, adult women and adult men. J Clin Endocrinol Metab 69:821-824 